\title{
Reconfiguring what is owed to patients in planning and delivering health care
}

The August issue of the Journal of Medical Ethics takes us from the intimate spaces of healthcare encounters between doctors, nurses and patients to the outer reaches of health policy design and planning. We travel from the early days of life with arguments that consider when children's participation in research should begin and end, to its very end - with considered reflections on how best to provide assistance in the dying process both within and outside the healthcare context. One striking feature of our journey through the various claims presented in this set of papers is a concerted and carefully reasoned attempt to reconfigure and clarify the precise nature of the duties that are owed to patients. This effort takes place both in the context of particular healthcare encounters, and in terms of how we conceive of, and plan, the delivery of health services in changing political times. Novel obligations, subtle interpretations of well-established duties, and new recommendations for innovation in practice abound as we map out the argumentative terrain presented to us by this month's contributors.

\section{ON WHAT IS OWED TO PATIENTS AT THE BEDSIDE}

Three papers in this month's issue focus our attention on what is owed by practitioners to patients at the bedside, and what interventions might best realise these duties in practice. Charles (see page 506) examines a broader relational conception of autonomy, which she believes better captures what is owed to patients receiving care in hospital, and provides a compelling case that, as expert nurses are best placed to support patient autonomy so long as they are properly supported by the institutions in which they work. For Charles, this emphasis on the consequent obligations that institutions possess in ensuring that nurses can act as they ought means that the moral agency of institutions is best understood as being derived from an account of enacting the moral agency of the practitioners who work within those institutions.
Bleese and colleagues (see page 549) are also concerned with the big-picture question of how practitioners' obligations can be delivered to patients in the round. However, their concern lies not within the relationship between practitioners and healthcare institutions, but with the relationship between practitioners and their knowledge and interpretation of particular diseases that are taken, within the profession and beyond, to be contested. Their particular concern is Chronic Fatigue Syndrome (CFS), and the extensive empirical data that suggests doctors and medical students dispute whether CFS is a real condition, and that this leads to patients with CFS experiencing the health encounter and the medicalisation of their condition negatively. The analysis presented targets an often under-recognised duty that doctors and other healthcare practitioners possess: to not expose their patients to epistemic injustice. For Bleese et al, the requirements of this duty require practitioners to adopt phenomenological tools that enable them to take steps to ensure that they do not deliberately or inadvertently marginalise their patients through denying or ignoring patients' own experiences, understandings, and conceptual accounts of their condition, and how it ought to be managed. One interesting and potentially fruitful avenue for further enquiry here would be to link these novel, if controversial, claims about the wrong of epistemic injustice with the normative arguments around consent to treatment, particularly in light of the legal and professional requirements to adopt more patient-centric approaches to obtaining informed consent that have emerged recently.

Woollard and Porter (see page 515) also tease out a concern that arises in healthcare encounters after birth: how the benefits of breastfeeding are presented to new mothers, with mothers' choices to bottle feed being understood and experienced as a failure of their maternal duties. At first glance, this looks to be another situation in which Bleese et al would recognise new mothers as being vulnerable to epistemic injustice in their healthcare encounters, but Woollard and Porter claim, instead, that the pressure to breastfeed that is expressed by healthcare practitioners is problematic in the sense that they have significantly misunderstood the demands of a different duty within their role: to support the mother in realising the correct understanding of the mother's duty to benefit her child.

Moving away from particular treatment encounters to end of life care practices, Bernheim and Raus (see page 489) and Hagens et al. (see page 543) offer two arguments for how assistance dying ought to be managed in relation to healthcare practice. Bernheim and Raus defend the view that an optimally functioning health system is one that integrates (where it is lawful) the practice of euthanasia into the delivery of well-established palliative care protocols. Drawing on the Belgian concepts of 'integral palliative care' and 'palliative futility', Bernheim and Raus's point is that essentialist arguments that seek to claim incompatibility between euthanasia and palliative care necessarily deny certain fundamental tenets that are embraced within the palliative care movement itself. These include the centring of empirical evidence about patients outcomes and experiences, a rejection of canonical definitions, and respect for a plurality of reasonable views concerning end of life decision-making pathways. Hagens et al, in contrast, take us across the border to the Netherlands to reflect on the established Dutch practice of demedicalised assistance in suicide (DAS). The authors present important qualitative data that reveals that patients seeking DAS had turned away from healthcare services due to dissatisfaction with the communication they had experienced when discussing their options with their doctors. This evidence gives rise to an argument that careful considerations of whether DAS is preferable to physician assistance in suicide, when the latter approach can, if provided in a timely manner, appear to be able to prevent harms that cannot be managed through a demedicalised approach. 


\section{ON WHAT IS OWED IN PLANNING HEALTH POLICY}

Turning our attention away from the patient-practitioner setting and towards the world of health policy, Rumbold (see page 501) argues against a recent interpretation by Daniel Engster that the 'specialness' of healthcare lies in its caring, rather than its purely health, dimensions. This account, Rumbold suggests, cannot differentiate health from most other sorts of goods, by virtue of its particular kind of moral significance. Notwithstanding these criticisms, Rumbold holds that the focus on care does allow health (and questions relating to its just distribution in health policy) to be understood as being concerned with the treatment of those who are already unwell - a morally significant feature that Rumbold believes could establish the foundation for a revised specialness theory of healthcare.

When conceptual work on aspects of healthcare is focused on the question of health interventions, further challenges to standard justifications for ethical policy-making arise. For those arguing against nudges (a certain kind of intervention that aims to encourage and enable optimal decision-making or behaviour without the use of force), a common objection is that this kind of practice undermines deliberative choice, thereby compromising autonomy. Levy (see page 495) scrutinises this argument through analysing a particular kind of nudge: a 'nudge to reason', which aims to make the individual who is nudged more responsive to genuine evidence - essentially the 'attempt to change behaviour by changing minds' (see page 495), as he describes it. While not all nudges fall into the 'nudge to reason' category, those that do are, he believes, entirely justifiable (and, potentially, ought to be prioritised in a political landscape in which there is evidence that weaknesses have arisen due to policy-makers' inability to respond appropriately to persuasive evidence).

Herlitz (see page 510) takes us squarely into what is perhaps the most common policy-level question that arises in medical ethical analysis: that of the fair allocation of medical resources. Given the basic relationship between wealth and life expectancy, Herlitz asks whether, on egalitarian grounds, we ought to weigh the benefits of alternative interventions on income-based grounds to ensure that the health benefits for the poor are prioritised. While he does not ultimately argue definitively for or against this position, the value of the paper lies in the important contribution that is made to our understanding about which empirical conditions would need to be proven in order to make such an argument persuasively. Health policy-making that aims for egalitarianism in prioritising investment in health interventions would need to take these relationships seriously, if income inequality is not merely going to be ignored as a morally relevant consideration in delivering equitable health outcomes.

Competing interests None declared.

Provenance and peer review Not commissioned; internally peer reviewed.

(C) Article author(s) (or their employer(s) unless otherwise stated in the text of the article) 2017. All rights reserved. No commercial use is permitted unless otherwise expressly granted. 